\title{
Corrigendum: Large-scale genome-wide association analysis of bipolar disorder identifies a new susceptibility locus near ODZ4
}

\author{
Psychiatric GWAS Consortium Bipolar Disorder Working Group \\ Nat. Genet. 43, 977-983 (2011); published online 18 September 2011; corrected after print 21 June 2012
}

In the version of this article initially published, there were errors in the consortium membership list and the associated affiliations and in the acknowledgements and contributions sections. These errors and their corrections are detailed below by section.

Consortium members:

Janice M. Fullerton was omitted from the membership list and has now been added with affiliations 76 and 77 . Phil H. Lee was listed incorrectly as Phil L. Hyoun. Fan Meng was listed incorrectly as Fan Guo Meng, and the associated affiliation has been changed from 51 to 54 . Robert Thompson was assigned affiliation 50; the correct affiliation is 54. Marian Hamshere and Valentina Moskvina were assigned affiliation 26; the correct affiliation for both is 22. Richard Day was assigned affiliation 47; the correct affiliation is 46. Jun Li was assigned affiliation 24; the correct affiliation is 48 . In addition to the affiliation originally listed for Sebastian Zöllner and Peng Zhang, both have now also been assigned affiliation 4. Howard Endenberg has now also been assigned affiliation 12, and Shaun Purcell has now also been assigned affiliation 1.

Consortium affiliations:

Affiliation 46 was originally given as the University of Dundee School of Medicine, Nethergate, Dundee, UK. The correct affiliation is the Division of Neuroscience, University of Dundee, Ninewells Hospital \& Medical School, Dundee, UK. Affiliation 47 was originally given as the School of Neurology, Neurobiology and Psychiatry, Royal Victoria Infirmary, Newcastle upon Tyne, UK. The correct affiliation is the Department of Human Genetics, University of Michigan, Ann Arbor, Michigan, USA. This affiliation was also listed out of order and has now been changed to affiliation 48. Affiliation 76 was originally given as the Prince of Wales Medical Institute, Sydney, Australia. The correct affiliation is Neuroscience Research Australia, Sydney, Australia.

Acknowledgments:

The Stanley Foundation for Medical Research was listed as a source of funding. The correct name is the Stanley Medical Research Institute, and the Merck Genome Research Institute has also been added as a source of support.

Contributions:

In four instances, the contribution of Sven Cichon was indicated with the incorrect spelling S. Chichon instead of S. Cichon. Manuel A. Ferreira (M.A.F.) was incorrectly listed as a contributor to manuscript preparation. Manuel A. Ferreira (M.A.F.) was listed as a contributor to primary study data at the NIMH/Pritzke; the correct contributor was Matthew Flickinger (M.F.). In the section listing contributors to replication data, Neuroscience Research Australia was named incorrectly as the Prince of Wales Medical Institute, and Janice M. Fullerton (J.M.F.) has been added as a contributor at this site and at the University of New South Wales.

The errors detailed above have been corrected in the HTML and PDF versions of the article. In addition, the subsections of the contributions section detailing the individuals contributing to primary study data and replication data were omitted from the original HTML version of the article, and this error has now been corrected.

\section{Erratum: BAP1 loss defines a new class of renal cell carcinoma}

Samuel Peña-Llopis, Silvia Vega-Rubín-de-Celis, Arnold Liao, Nan Leng, Andrea Pavía-Jiménez, Shanshan Wang, Toshinari Yamasaki, Leah Zhrebker, Sharanya Sivanand, Patrick Spence, Lisa Kinch, Tina Hambuch, Suneer Jain, Yair Lotan, Vitaly Margulis, Arthur I Sagalowsky, Pia Banerji Summerour, Wareef Kabbani, S W Wendy Wong, Nick Grishin, Marc Laurent, Xian-Jin Xie, Christian D Haudenschild, Mark T Ross, David R Bentley, Payal Kapur \& James Brugarolas

Nat. Genet. 44, 751-759 (2012); published online 10 June 2012; corrected after print 21 June 2012

In the version of this article initially published, the $\mathrm{P}$ value given in the abstract for the anticorrelation between $B A P 1$ and $P B R M 1$ mutations was given incorrectly as $9 \times 10^{-6}$ instead of $3 \times 10^{-5}$. The definition of mutant allele ratios (MARs) in the text on p. 1 of the PDF has been corrected. The titles and legends of Figure 1 and Table 1 incorrectly stated that data were presented from multiple tumors and tumorgrafts; these have been corrected to reflect that data came from one index subject. The title of Figure 2 originally referred to mutated residues; this has now been corrected to state that alterations in BAP1 are shown. On p. 7 of the PDF the text has been corrected to state that "a few tumors had loss of both BAP1 and PBRM," whereas the text originally incorrectly cited somatic mutations in both genes. The errors have been corrected in the HTML and PDF versions of the article.

\section{Erratum: The Pediatric Cancer Genome Project}

James R Downing, Richard K Wilson, Jinghui Zhang, Elaine R Mardis, Ching-Hon Pui, Li Ding, Timothy J Ley \& William E Evans Nat. Genet. 44, 619-622 (2012); published online 29 May 2012; corrected after print 9 July 2012

In the version of this article initially published, there were errors in the labeling of Figure 1b. Specifically, hyperdiploidy was mislabeled twice as hypodiploidy, and hypodiploidy was defined incorrectly as $>44$ instead of $<44$ chromosomes. These errors have been corrected in the HTML and PDF versions of the article. 